\title{
Structural modeling of the flagellum MS ring protein FliF reveals similarities to the type III secretion system and sporulation complex
}

Julien R Bergeron

The flagellum is a large proteinaceous organelle found at the surface of many bacteria, whose primary role is to allow motility through the rotation of a long extracellular filament. It is an essential virulence factor in many pathogenic species, and is also a priming component in the formation of antibiotic-resistant biofilms. The flagellum consists of the export apparatus and rotor on the cytosolic side; the basal body, spanning the bacterial membrane(s) and periplasm; and the hook-filament, that protrudes away from the bacterial surface. Formation of the basal body MS ring region, constituted of multiple copies of the protein FliF, is one of the initial steps of flagellum assembly. However, the precise architecture of FliF is poorly understood. Here, I report a bioinformatics analysis of the FliF sequence from various bacterial species, suggesting that its periplasmic region is composed of three globular domains. The first two are homologous to that of the type III secretion system injectisome proteins Sct), and the third possesses a similar fold to that of the sporulation complex component SpollIAG. I also describe that Chlamydia possesses an unusual FliF protein, lacking part of the SctJ homology domain and the SpolllAG-like domain, and fused to the rotor component FliG at its C-terminus. Finally, I have combined the sequence analysis of FliF with the EM map of the MS ring, to propose the first atomic model for the FliF oligomer, suggesting that FliF is structurally akin to a fusion of the two injectisome components SctJ and SctD. These results further define the relationship between the flagellum, injectisome and sporulation complex, and will facilitate future structural characterization of the flagellum basal body. 


\section{Structural modeling of the flagellum MS ring protein FliF reveals}

2 similarities to the type III secretion system and sporulation complex.

3

4

5

6 Julien R. C. Bergeron

7

8 Department of Biochemistry and Molecular Biology, and Centre for Blood Research,

9 University of British Columbia, Vancouver, Canada

10

11 Current address: Department of Biochemistry, University of Washington, Seattle, USA

12

13

14 Correspondence to:

15 Julien R. C. Bergeron

16 University of Washington

171959 NE Pacific Street

18 Box 357350

19 Seattle, WA 98195

20 Email: jrcberge@uw.edu 


\section{ABSTRACT:}

22 The flagellum is a large proteinaceous organelle found at the surface of many bacteria, whose 23 primary role is to allow motility through the rotation of a long extracellular filament. It is an 24 essential virulence factor in many pathogenic species, and is also a priming component in the 25 formation of antibiotic-resistant biofilms. The flagellum consists of the export apparatus and 26 rotor on the cytosolic side; the basal body, spanning the bacterial membrane(s) and periplasm; 27 and the hook-filament, that protrudes away from the bacterial surface. Formation of the basal 28 body MS ring region, constituted of multiple copies of the protein FliF, is one of the initial steps 29 of flagellum assembly. However, the precise architecture of FliF is poorly understood. Here, I report a bioinformatics analysis of the FliF sequence from various bacterial species, suggesting

31 that its periplasmic region is composed of three globular domains. The first two are homologous 32 to that of the type III secretion system injectisome proteins SctJ, and the third possesses a similar 33 fold to that of the sporulation complex component SpoIIIAG. I also describe that Chlamydia 34 possesses an unusual FliF protein, lacking part of the SctJ homology domain and the SpoIIIAG35 like domain, and fused to the rotor component FliG at its C-terminus. Finally, I have combined the sequence analysis of FliF with the EM map of the MS ring, to propose the first atomic model for the FliF oligomer, suggesting that FliF is structurally akin to a fusion of the two injectisome components SctJ and SctD. These results further define the relationship between the flagellum, injectisome and sporulation complex, and will facilitate future structural characterization of the 40 flagellum basal body. 


\section{INTRODUCTION}

43 Bacteria interact with their environment using a range of surface appendages, including flagella, 44 pili, fimbriae, and secretion systems (Fronzes, Remaut et al. 2008). In particular, the flagellum is 45 responsible for motility in many bacteria (Terashima, Kojima et al. 2008), but it is also frequently associated with adhesion to surfaces and/or other cells (Belas 2014). Flagella are found in many bacterial families, including most gram-positive, proteobacteria and spirochetes (Chen, Beeby et al. 2011, Minamino and Imada 2015). Notably, it is an essential virulence factor in many pathogenic species, such as Salmonella, E. coli, Clostridium, Pseudomonas, Helicobacter, Vibrio, Burkholderia, and Campylobacter, making the flagellum a potential target for new antibacterial therapeutics (Duan, Zhou et al. 2013).

The bacterial flagellum is constituted of four distinct regions (Stock, Namba et al. 2012, Morimoto and Minamino 2014, Minamino and Imada 2015) (Figure 1A). On the cytosolic side and anchored to the inner-membrane, the type III secretion system (T3SS) apparatus and the rotor are responsible for the secretion of the extracellular components and rotation of the system, respectively. The membrane-embedded structure that traverses the cytoplasmic membrane and periplasmic space (as well as the outer membrane for gram-negative bacteria) is called the basal body. The hook is a curved filament, $\sim 55 \mathrm{~nm}$ in length (for the prototypical Salmonella typhimurium flagellum), that protrudes away from the basal body. It is prolonged by the filament, a long structure ( $>10 \mu \mathrm{m}$ in $S$. typhimurium) responsible for motility and adherence.

Genetic studies have revealed that the inner-membrane protein FliF forms a two ring-shaped structures called the MS rings, that assembles early during flagellum morphogenesis (Kubori, Shimamoto et al. 1992), likely around elements of the T3SS. This in turn recruits the rotor and ATPase in the cytosol, leading to a secretion-competent complex, which can export the hook protein (and other flagellar components) to the extracellular environment through its central pore. A subsequent substrate specificity switching event, controlled by elements of the T3SS, lead to the assembly of the filament (Minamino, Imada et al. 2008). FliF is an $\sim 60 \mathrm{kDa}$ protein, localized to the inner-membrane through the Sec pathway. Sequence analysis has indicated that it possesses two transmembrane helices, flanking a large periplasmic region (Ueno, Oosawa et al. 1994). At the C-terminus, a cytosolic peptide has been shown to interact with FliG (Levenson, Zhou et al. 2012, Ogawa, Abe-Yoshizumi et al. 2015), a 
73 forming the MS ring oligomer (Suzuki, Yonekura et al. 2004), although analyses of intact 74 flagellum particles have identified 24-, 25- and 26-fold symmetry for this region of the basal 75 body (Thomas, Francis et al. 2006).

76 A number of components from the cytosolic export apparatus are homologous to that of the 77 injectisome (Figure 1B), another bacterial complex whose role is to inject so-called "effector" 78 proteins inside the cytosol of target or symbiotic cells (Buttner 2012, Diepold and Armitage 2015). Indeed, phylogenetic studies have revealed that the flagellum export apparatus is likely

80 the evolutionary ancestor of the injectisome (Abby and Rocha 2012), with both complexes 81 employing a similar T3SS for protein export and coordinated assembly (Buttner 2012, Diepold 82 and Armitage 2015).

83 In particular, FliF shows significantly sequence similarity to an inner-membrane component of 84 the injectisome, SctJ (24\% sequence identity with the Salmonella homologue, $22 \%$ sequence identity with the EPEC homologue, for residues 52-217 of FliF) (Ueno, Oosawa et al. 1994). 86 SctJ forms a 24-mer ring structure in the inner-membrane, similar to that of FliF, and structural characterization have revealed the molecular details of its architecture and oligomerization (Yip, Kimbrough et al. 2005, Bergeron, Worrall et al. 2013, Bergeron, Worrall et al. 2015). Specifically, the periplasmic region of SctJ consists of two globular domains with a canonical "ring building motif" (RBM) fold (Crepin, Prasannan et al. 2005, Yip, Kimbrough et al. 2005, Spreter, Yip et al. 2009), found in several oligomeric proteins. The two RBMs are joined by a rigid linker, which was shown to promote oligomerization (Bergeron, Worrall et al. 2015).

93 Recently, two proteins essential for the sporulation process in Bacillus subtilis, SpoIIIAH and 94 SpoIIIAG, were shown to be homologous to SctJ and FliF (Camp and Losick 2008, Meisner, 95 Wang et al. 2008). This led to the suggestion that these proteins are part of a complex that directs 96 the transport of proteins and/or nutrients between the mother cell and the endospore (Figure 1C), 97 although such complex has not been observed directly (Crawshaw, Serrano et al. 2014).

98 There is currently very little data on the architecture of FliF, and its relationship to the 99 injectisome component SctJ and the sporulation complex components SpoIIIAH and SpoIIIAG. 100 In this study, I have exploited the recent structural studies of the injectisome and sporulation 101 complex, in order to update our understanding of the FliF architecture using computational and 102 bioinformatics analyses. Based on these, I propose that the periplasmic region of FliF consists of 103 a SctJ homology domain, as well as a FliF-specific domain structurally homologous to the 
104 sporulation complex component SpoIIIAG. I also report that in the Chlamydiacae family, the

105 FliF protein differs significantly from other species, as it lacks part of the SctJ homology region,

106 and is fused to a FliG-like domain at the C-terminal cytosolic end. Finally, I have combined

107 previously determined EM maps and structural modeling to introduce the first molecular model

108 for the FliF periplasmic region, suggesting that FliF is akin to a fusion of the injectisome basal

109 body inner-membrane components SctJ and SctD. This unexpected observation has implications

110 in the understanding of the evolutionary relationship between the flagellum, injectisome and

111 sporulation complex.

112

\section{MATERIALS AND METHODS:}

\section{Sequence mining, analysis and alignment:}

115 All protein sequences were identified with the National Centre for Biotechnology Information

116 protein database RefSeq (Pruitt, Tatusova et al. 2012). Multiple sequence alignments were

117 generated with ClustalW (McWilliam, Li et al. 2013) using default parameters. For secondary

118 structure-based multiple alignments, a composite alignment was generated manually based on

119 the individual pairwise alignments. Alignment figures were produced with ESPript (Gouet, 120 Robert et al. 2003).

121 Secondary structure elements, signal sequences, transmembrane helices and structural

122 homologues were predicted with the PSIPRED server (Buchan, Minneci et al. 2013). Protein

123 sequence identity was calculated with the Needleman-Wunsch algorithm on the EBI server (Li,

124 Cowley et al. 2015), using default parameters. Signal sequences were predicted with SignalP 4.1

125 (Petersen, Brunak et al. 2011).

126

127 Modeling and refinement:

128 Structure-based alignment and initial models were obtained with Phyre (Kelley, Mezulis et al.

129 2015), and the models were further refined by performing 1000 cycles of the Relax procedure

130 (Rohl, Strauss et al. 2004) in Rosetta 3.4 (Leaver-Fay, Tyka et al. 2011), using the following

131 flags:

132 -database $\sim$ rosetta/rosetta_database

133 -in:file:s input.pdb

134 -in:file:fullatom 
$135 \quad-$ relax:thorough

136 -nstruct 1000

137 -out:file:silent relax.silent

138 The geometry of the obtained models was analyzed with the PSVS suite (Bhattacharya, Tejero et 139 al. 2007).

140

141 EM map docking and symmetry modeling:

142 The flagellum, injectisome, and FliF EM maps (EMDB ID 1887, 1875 and personal

143 communication from K. Namba), were docked with the MatchMaker tool in Chimera (Goddard, 144 Huang et al. 2007). Models of the FliF RBMs were placed in their putative location of the FliF 145 EM map manually using Chimera.

146 For the EM-guided symmetry modeling, 24-fold, 25-fold and 26-fold symmetry definition files 147 were generated, and used for the rigid-body step of the EM-guided symmetry modeling 148 procedure described previously (Bergeron, Worrall et al. 2013). Briefly, the individual domains 149 were manually placed in the corresponding region of the EM map, and 1000 rigid-body decoy 150 models were generated with imposed symmetry and with a restraint for fit into the FliF EM map 151 density, at $22 \AA$ resolution. The obtained models were isolated with the Cluster procedure in 152 Rosetta, and scores calculated with the Score procedure using the lowest-energy model as a 153 template for RMS calculations.

154 The flags used for the modeling procedure are listed in the Supplementary Methods section.

156 RESULTS AND DISCUSSION

\section{Characterization of the FliF domain organization}

158 In order to identify conserved features, I gathered FliF sequences from a number of human 159 pathogens, spanning gram-negative, gram-positive and spirochete bacteria. Most of the FliF 160 sequences are similar in length ( 560 amino acids), but show limited sequence conservation 161 (Table 1). I next used sequence analysis servers to predict secondary structure elements and other 162 structural and functional features. Two transmembrane (TM) helices are predicted, between 163 residues 20-45 and residues 445-470, and a secretion signal peptide targeting it for secretion and 164 inner-membrane localization (Kudva, Denks et al. 2013) is predicted at the N-terminus. All FliF 165 sequences show a very similar secondary sequence prediction pattern (Supplementary figure 1), 
166 and similarity to SctJ was identified for residues 50-220 in all sequences (hereafter referred to as

167 the SctJ homology domain), but no structural homologues were found for residues 220-440

168 (FliF-specific domain). Within this region, residues 305-360 of the FliF-specific domain are

169 predicted unstructured in all sequences.

170 I then generated a multiple alignment of all FliF sequences, and used it to map the predicted 171 secondary structure and identified domains (Figure 2). This further illustrates the conserved

172 domain organization in all FliF orthologues. The two RBMs of the SctJ homology domain 173 (labeled RBM1 and RBM2) are well conserved, as is the linker L1 between these. It has been 174 shown that in SctJ this linker plays a role in ring assembly (Bergeron, Worrall et al. 2015), 175 suggesting that this may also the case in FliF. In contrast, the linker region L2, separating the 176 SctJ homology domain to the FliF-specific domain, is highly variable.

177

\section{The Chlamydiacae FliF-FliG fusion protein}

179 While the FliF domain organization described above was found in most FliF sequences, one 180 notable exception was identified, for the Chlamydiaceae family, where the FliF sequence is 181 notably shorter ( $\sim 330$ amino acids). Sequence analysis and multiple sequence alignment from all 182 available Chlamydiaceae homologues (Figure 3A) revealed that the $\mathrm{N}$-terminal signal sequence 183 and the two TM (residues 16-33 and 250-275) are present, but the periplasmic region is 184 significantly shorter. Residues 60-145 show some sequence similarity to SctJ, but only 185 encompassing RBM2. No structural homologues could be identified for residues 165-235, 186 however the predicted secondary structure matches that of the canonical RBM.

187 In most orthologues, the C-terminus cytosolic region of FliF (residues 520-560) binds to the 188 protein FliG (Levenson, Zhou et al. 2012, Ogawa, Abe-Yoshizumi et al. 2015), a 37 KDa 189 protein possessing three domains, labeled N, M and C. FliF interacts with domain N, while both $190 \mathrm{M}$ and $\mathrm{C}$ bind to the rotor (also known as C-ring) component FliM (Brown, Terrazas et al. 2007, 191 Minamino, Imada et al. 2011), as illustrated on figure 3B. However, a sequence similarity search 192 revealed that in the Chlamydia FliF, the cytosolic region is actually homologous to the M region 193 of FliG (not shown), revealing that in this species the protein is in fact a FliF-FliG fusion. 194 Chlamydia is not thought be a flagellated bacterium, and possesses only a few flagellar genes, 195 namely FliF, FliL and FlhA. It does however possess a functional injectisome that is essential for 196 virulence (Peters, Wilson et al. 2007), and it has been shown that Chlamdia flagellar proteins 
197 interact with components of its injectisome (Stone, Bulir et al. 2010). FliM (and FliN, another C-

198 ring component) is homologous to the Chlamydia injectisome component SctQ (Notti,

199 Bhattacharya et al. 2015). It is therefore possible that the FliF-FliG fusion interacts with SctQ

200 (Figure 3B). While the Chlamydia injectisome possesses both SctJ and SctD homologues(Nans,

201 Kudryashev et al. 2015), expression of fliF was shown to be concomitant with that of the

202 injectisome (Hefty and Stephens 2007). It This remains to be tested experimentally if FliF is

203 included in the Chlamydia injectisome.

204 Interestingly, a FliF-FliG fusion is not entirely unprecedented, as two artificial FliF-FliG fusions

205 have been reported in S. typhimurium (Francis, Irikura et al. 1992, Thomas, Morgan et al. 2001).

206 In both cases, the fusion does not impair flagellum assembly and rotation, but induces a bias in

207 the rotation direction. Since it is not yet clear if the Chlamydia FliF-FliG fusion protein is part of

208 a proto-flagellum, or contributes to the injectisome, the implications for this observation is not

209 clear, but suggests that such a fusion protein may be functional.

210

\section{Structural modeling of the SctJ homology domain}

212 Structures of the periplasmic domains of both SctJ from EPEC (Crepin, Prasannan et al. 2005,

213 Yip, Kimbrough et al. 2005) and S. typhimurium (Bergeron, Worrall et al. 2015) have been

214 reported. Exploiting this information, a structural model for FliF was generated using the

215 prototypical S. typhimurium FliF sequence. Despite the predicted structural homology, the

216 sequence conservation between FliF and SctJ is low (Figure 4A). I therefore employed a

217 secondary structure alignment-based procedure for modeling the SctJ homology domain,

218 spanning residues 50 to 221 . However, the relative orientation of the two RBMs in FliF is not

219 known, and may differ from that of SctJ. I therefore modeled the two RBMs independently, and

220 refined the obtained models with Rosetta (see Materials and Methods for details). As shown on

221 figure 4B and 4C, both RBMs converged to a local energy minimum within an RMSD of $1 \AA$ to

222 the lowest-energy model in the refinement procedure. The models possess good geometry (table

223 2), and their overall architecture (Figure 4D and 4E) is expectedly similar to that of SctJ.

224

225 The FliF-specific domain is a RBM

226 I next sought to generate a structural model for the FliF-specific domain (residues 228-443). As

227 mentioned above, secondary structure prediction indicated that residues 228-309 and residues 
228 356-443 possess defined secondary structure, while residues 310-355 are predicted as 229 intrinsically disordered (Fig 2). I therefore hypothesized that the FliF-specific region consists of 230 two globular domains (D1 and D2) separated by a flexible linker. I then attempted to identify 231 structural homologues to these two regions, using the Threading server PsiPred (Buchan, 232 Minneci et al. 2013). Surprisingly, D1 is predicted to have structural homology to SctJ, although 233 only for the first two secondary structure elements (residues 229-268, Supplementary figure 2A).

234 Similarly, D2 was identified to have structural homology to the sporulation complex protein 235 SpoIIIAH, which also possesses a RBM fold and has been proposed to oligomerize into ring 236 structures (Levdikov, Blagova et al. 2012, Meisner, Maehigashi et al. 2012) (Supplementary 237 figure 2B). However, the structural homology was limited to the last three secondary structure 238 elements (residues 386-436). Based on these observations, I postulated that the FliF-specific 239 domain is a "split" RBM that possesses a large insert in the loop between the first and second 240 strands (Figure 5A). A secondary structure similarity search for the FliF-specific domain with 241 this insert removed $\left(\mathrm{FliF}_{228-443} \Delta 274-378\right)$ confirmed overall fold similarity to both SctJ and 242 SpoIIIAH (Supplementary figure 2C).

243 A "split" RBM is not unprecedented, as it is also predicted in the sporulation complex 244 component SpoIIIAG (figure 5A). In both proteins an insert is predicted between the first and 245 second strand of the RBM, with to four putative $\beta$-strands in the inserted domain. This insert is 246 large enough to accommodate a small globular domain; alternatively, it is possible that the insert 247 adopts an extended loop conformation stabilized by $\beta$-strands.

248 In order to build an atomic model for the FliF RBM3, the SctJ-derived model for FliF $230-275$ was 249 combined with the SpoIIIAH-derived model for FliF $380-440$, and the resulting structural model 250 was refined with Rosetta. As shown on figure 5B, the RMSD to the lowest energy model is 251 significantly higher than for the RBM1 and RBM2 models (around $3.5 \AA$ for most decoys), 252 which is perhaps expected, as the starting model was a presumably poorer, composite model. 253 Nevertheless there is a clear energy funnel, indicative that the modeling procedure is converging. 254 The obtained FliF RBM3 model, shown on figure 5C, possesses the canonical RBM fold, with 255 good overall geometry (Table 2) and an elongated architecture similar to the RBM2 of SctJ and 256 SpoIIIAH, with the insert between strands 1 and 2 located on one side of the structure.

\section{Localization of the FliF domains}


259 Previous EM studies have shown that in isolation, FliF forms a doughnut-shaped oligomer with 260 two side rings corresponding to the MS rings seen in the intact basal body (Suzuki, Yonekura et 261 al. 1998, Thomas, Francis et al. 2006). The FliG-binding domain forms the cytoplasmic M ring, 262 but little is known about the organization of the periplasmic domains. However, the organization 263 of the injectisome basal body, and that of SctJ in particular, is well characterized (Schraidt and 264 Marlovits 2011, Bergeron, Worrall et al. 2013, Bergeron, Worrall et al. 2015). By comparing the 265 EM reconstructions of the flagellar (Thomas, Francis et al. 2006) and injectisome (Schraidt and 266 Marlovits 2011) basal body complexes, localization of the FliF RBMs can be inferred. As shown on figure 6A, density attributed to the two globular domains of SctJ has clear equivalence in the flagellum, suggesting this location corresponds to the SctJ homology domain of FliF. EM map density for the flagellum S-ring corresponds to the injectisome protein SctD localization, and is also present in purified FliF (Suzuki, Yonekura et al. 1998), confirming that it is not attributed to another flagellar component. The periplasmic region of SctD is composed of three domains with an RBM fold (Spreter, Yip et al. 2009, Bergeron, Worrall et al. 2013), including the region corresponding to FliF density, suggesting that in the flagellum, this density can be attributed to the FliF-specific domain, which also possesses an RBM fold. Based on these observations, I propose that FliF is akin to a SctJ-SctD fusion (figure 6B), including the two RBMs of SctJ and its C-terminal TM helix, and the N-terminal TM and first RBM from SctD. This likely explains a major difference between flagellar basal body, where FliF assembles on its own in the inner membrane, and injectisome basal body, where coexpression of SctJ and SctD is required (Kimbrough and Miller 2000). Further sequence analysis will be required to decipher the exact evolutionary pathway leading to the emergence of two genes in the injectisome. It is however interesting to note that in the ancestral Myxococcus injectisome, the SctD homologue lacks a periplasmic domain, and only consists in the cytoplasmic FHA-fold domain found in all SctD homologues, (Bergeron, Worrall et al. 2013) followed by a predicted transmembrane domain. It is therefore likely that this gene was fused to a FliF duplication gene in subsequent injectisomes, leading to the SctD homologue including the FHA cytosolic domain and the three BRMs in the periplasm.

287 One can wonder how having two separate proteins is beneficial for the injectisome. Having two separate genes may allow for a more dynamic assembly/disassembly process. Indeed, most injectisomes are only transiently functional, and while the regulation of injectisome genes 
290 transcription is well characterized, the fate of the injectisome complex after its required function

291 (such as, for example, the SPI-1 injectisome of S. typhimurium after insertion in the Salmonella-

292 Containing Vacuole) is not known. It is possible that having two proteins allow for disassembly

293 of the basal body and recycling of the corresponding proteins (Diepold and Wagner 2014).

294 It is also interesting to note that SctD is the only inner-membrane component of the injectisome

295 and/or flagellum lacking a secretion signal. Indeed, deletion of the complete N-terminus of the $S$.

296 typhimurium StcD homologue allows the formation of intact basal body complexes (Bergeron,

297 Worrall et al. 2013). It remains to be shown how the periplasmic domain of this protein is

298 translocated across the inner membrane, but co-export with SctJ is an intriguing possibility, and

299 to my knowledge such co-secretion via the Sec pathway (through which both FliF and SctJ are

300 thought to be translocated) is unprecedented.

301

302 Modeling of the FliF oligomer

303 I next exploited the domain localization proposed above, the structural models of the three FliF

304 RBMs, and the previously determined EM map (Suzuki, Yonekura et al. 2004), to generate a

305 structural model of the FliF monomer. To that end, I positioned all three domains so that their

306 termini point towards the correct region of the map, and fitted into the EM map density with

307 Chimera (Goddard, Huang et al. 2007). Figure 7A shows that RBM1 is located near the inner-

308 membrane region, while RBM2 forms the neck of the structure. Finally, the RBM3 is located in

309 the region of density forming the S-ring. The insert in RBM3 points towards the lumen of the

310 ring, and could correspond to the gate density observed in the FliF EM structure (Suzuki,

311 Yonekura et al. 1998, Suzuki, Yonekura et al. 2004).

312 I next attempted to generate a model for the FliF oligomer. I applied the previously described

313 EM-guided modeling procedure (Bergeron, Worrall et al. 2013) to all three domains individually,

314 using the FliF map as a restraint, and applying 24-, 25- or 26-fold symmetry. In most cases the

315 procedure led to ring models that were too large for the EM map (not shown). This is

316 unsurprising, as it had been reported previously that the procedure requires experimental

317 structures (as opposed to homology models) to converge (Bergeron, Worrall et al. 2013). The

318 one exception was the modeling of the RBM3, using 25-fold symmetry. This procedure

319 generated three distinct clusters of models with clear energy funnels and good fit to EM density

320 (Figure 7B). Close inspection of the clusters reveals that they correspond to similar 
321 oligomerization modes and use the same interface, but with a slightly different angle between the

322 subunits (Figure 7C). I therefore exploited this 25-mer mode for the RBM3 to generate a 323 complete 25-mer model for FliF. The corresponding model, shown in figure 7D and included in

324 the supplementary material, matches well with the cryo-EM structure, with only a few loop 325 regions of RBM2 and RBM3 located outside of the density.

326 I emphasize that while only 25-fold symmetry led to a convergent model for RBM3, this should 327 not be considered as evidence that FliF forms a 25-mer. Indeed, the limited amount of data used 328 in the modeling procedure is not sufficient to generate an accurate structural model, and at the 329 resolution of the EM map ( $24 \AA)$ the difference between 25 and 26 subunits is indistinguishable.

330 It is also possible that the overall diameter of the complex appears smaller due to a small error in

331 the pixel size of the detector used, thus biasing the modeling process towards a lower oligomeric 332 state. However, the converging energy landscape for RBM3, and fit to EM density for all three 333 domains, suggest that the reported model may be exploiting the native general orientation and 334 oligomerization interface for all three domains. Additional experimental data, including 335 experimentally determined RBM1, RBM2 and RBM3 atomic structures, as well as a higher 336 resolution EM map of FliF and of the intact flagellum basal body, will be required to further 337 refine the FliF model.

338 Despite these significant limitations, several aspects of the FliF model are worth commenting on.

339 Firstly, the oblong shape of RBM2 forms an angle close to perpendicular to the membrane plane

340 (Figure 7A). In contrast, this domain is almost to parallel to the membrane plane in SctJ (Yip, 341 Kimbrough et al. 2005, Schraidt and Marlovits 2011, Bergeron, Worrall et al. 2013). While the 342 proposed orientation of FliF RBM2 is driven by the EM map density of the isolated FliF 343 oligomer, it is possible that it may differ in the intact flagellum basal body, and correspond to a 344 structural rearrangement upon recruitment of other flagellar components (rod and/or LP ring).

345 Higher resolution EM reconstruction of the full flagellum basal body will be required to identify 346 structural rearrangements associated with flagellar assembly.

347 It is similarly noteworthy that in the FliF model, RBM2 is located approximately $20 \AA$ away 348 from RBM1, unlike SctJ where the two domains form a direct interaction (Bergeron, Worrall et 349 al. 2015). The later domain arrangement was shown to be mediated by the linker region, with in 350 particular a conserved phenylalanine residue playing essential role in oligomerization (Phe 72 in 351 the S. typhimurium SctJ homologue PrgK). Interestingly, the linker between RBM1 and RBM2 is 
352 also well conserved in FliF (linker L1 in figure 2), with in particular a Phe found in most 353 orthologues at position 121 (and replaced with a different large hydrophobic residue in some 354 species), which could perform a similar role. It is therefore likely that this linker plays a role on 355 FliF assembly.

356 Finally, it is interesting to note that in the FliF model, RBM1 and RBM3 are in close proximity, 357 and would likely form direct interactions. Considering the limitations of the modeling procedure 358 described above, it would be premature to use this model to identify residues that are part of the 359 interaction interface. Nonetheless, continuous density in this region of the injectisome EM map 360 (Schraidt and Marlovits 2011) suggests that a direct interaction does exist between these two 361 domains, thus supporting a similar interaction in FliF.

\section{CONCLUSION:}

364 In this study I have presented evidence that the periplasmic region of most FliF orthologues consists of three globular domains possessing the canonical RBM motif. One exception is the Chlamydia FliF paralogue, which possesses only two of these, and has a FliG domain fusion at its C-terminus. By comparison with the injectisome basal body, I also propose the novel concept that FliF is akin to a fusion of SctJ and SctD. Finally I have combined this information to propose a model for the oligomeric arrangement of the periplasmic region of FliF. Further experimental validation will be required to confirm these observations, and to refine the FliF model.

372 These results shed new lights on the architecture and evolution of the flagellum MS ring.

373 Specifically, the domain organization of FliF highlights similarities with the injectisome, but also with the bacterial sporulation complex. The proposed concept that FliF corresponds to a fusion of SctJ and SctD likely explains why FliF can assemble spontaneously, while SctJ and SctD require co-expression. In addition, the identification of a Chlamydia FliF-FliG fusion suggests that this may correspond to an ancestral complex.

\section{ACKNOWLEDGEMENTS:}

380 I am grateful to Dr Keiichi Namba and Dr Hirofumi Suzuki for providing the FliF EM map. I thank Dr Natalie Zeytuni and Dr Morgan Bye for critical comments on the manuscript. 
384

385

386

387

388

389

390

391

392

393

394

395

396

397

398

399

400

401

402

403

404

405

406

407

408

409

410

411

412

413

414

415

416

417

418

419

420

421

422

423

424

425

426

\section{REFERENCES:}

Abby, S. S. and E. P. Rocha (2012). "The non-flagellar type III secretion system evolved from the bacterial flagellum and diversified into host-cell adapted systems." PLoS Genet 8(9): e1002983.

Belas, R. (2014). "Biofilms, flagella, and mechanosensing of surfaces by bacteria." Trends Microbiol 22(9): 517-527.

Bergeron, J. R., L. J. Worrall, S. De, N. G. Sgourakis, A. H. Cheung, E. Lameignere, M. Okon, G. A. Wasney, D. Baker, L. P. McIntosh and N. C. Strynadka (2015). "The modular structure of the inner-membrane ring component PrgK facilitates assembly of the type III secretion system basal body." Structure 23(1): 161-172.

Bergeron, J. R., L. J. Worrall, N. G. Sgourakis, F. DiMaio, R. A. Pfuetzner, H. B. Felise, M. Vuckovic, A. C. Yu, S. I. Miller, D. Baker and N. C. Strynadka (2013). "A refined model of the prototypical Salmonella SPI-1 T3SS basal body reveals the molecular basis for its assembly." PLoS Pathog 9(4): e1003307.

Bhattacharya, A., R. Tejero and G. T. Montelione (2007). "Evaluating protein structures determined by structural genomics consortia." Proteins 66(4): 778-795.

Brown, P. N., M. Terrazas, K. Paul and D. F. Blair (2007). "Mutational analysis of the flagellar protein FliG: sites of interaction with FliM and implications for organization of the switch complex." L Bacteriol 189(2): 305-312.

Buchan, D. W., F. Minneci, T. C. Nugent, K. Bryson and D. T. Jones (2013). "Scalable web services for the PSIPRED Protein Analysis Workbench." Nucleic Acids Res 41(Web Server issue): W349-357.

Buttner, D. (2012). "Protein export according to schedule: architecture, assembly, and regulation of type III secretion systems from plant- and animal-pathogenic bacteria." Microbiol Mol Biol Rev 76(2): 262-310.

Camp, A. H. and R. Losick (2008). "A novel pathway of intercellular signalling in Bacillus subtilis involves a protein with similarity to a component of type III secretion channels." Mol Microbiol 69(2): 402-417.

Chen, S., M. Beeby, G. E. Murphy, J. R. Leadbetter, D. R. Hendrixson, A. Briegel, Z. Li, J. Shi, E. I. Tocheva, A. Muller, M. J. Dobro and G. J. Jensen (2011). "Structural diversity of bacterial flagellar motors." EMBO I 30(14): 2972-2981.

Crawshaw, A. D., M. Serrano, W. A. Stanley, A. O. Henriques and P. S. Salgado (2014). "A mother cell-to-forespore channel: current understanding and future challenges." FEMS Microbiol Lett 358(2): 129-136.

Crepin, V. F., S. Prasannan, R. K. Shaw, R. K. Wilson, E. Creasey, C. M. Abe, S. Knutton, G. Frankel and S. Matthews (2005). "Structural and functional studies of the enteropathogenic Escherichia coli type III needle complex protein EscJ." Mol Microbiol 55(6): 1658-1670. Diepold, A. and J. P. Armitage (2015). "Type III secretion systems: the bacterial flagellum and the injectisome." Philos Trans R Soc Lond B Biol Sci 370(1679).

Diepold, A. and S. Wagner (2014). "Assembly of the bacterial type III secretion machinery." FEMS Microbiol Rev 38(4): 802-822.

Duan, Q., M. Zhou, L. Zhu and G. Zhu (2013). "Flagella and bacterial pathogenicity." L Basic Microbiol 53(1): 1-8. 
427 Francis, N. R., V. M. Irikura, S. Yamaguchi, D. J. DeRosier and R. M. Macnab (1992). 428 "Localization of the Salmonella typhimurium flagellar switch protein FliG to the 429 cytoplasmic M-ring face of the basal body." Proc Natl Acad Sci U S A 89(14): 6304-6308.

430 Fronzes, R., H. Remaut and G. Waksman (2008). "Architectures and biogenesis of non431 flagellar protein appendages in Gram-negative bacteria." EMBO J 27(17): 2271-2280.

432 Goddard, T. D., C. C. Huang and T. E. Ferrin (2007). "Visualizing density maps with UCSF 433 Chimera." I Struct Biol 157(1): 281-287.

434 Gouet, P., X. Robert and E. Courcelle (2003). "ESPript/ENDscript: Extracting and rendering 435 sequence and 3D information from atomic structures of proteins." Nucleic Acids Res 31(13): 3320-3323.

437 Hefty, P. S. and R. S. Stephens (2007). "Chlamydial type III secretion system is encoded on 438 ten operons preceded by sigma 70-like promoter elements." L Bacteriol 189(1): 198-206.

439 Kelley, L. A., S. Mezulis, C. M. Yates, M. N. Wass and M. J. Sternberg (2015). "The Phyre2 web 440 portal for protein modeling, prediction and analysis." Nat Protoc 10(6): 845-858.

441 Kimbrough, T. G. and S. I. Miller (2000). "Contribution of Salmonella typhimurium type III 442 secretion components to needle complex formation." Proc Natl Acad Sci U S A 97(20): 443 11008-11013.

444 Kubori, T., N. Shimamoto, S. Yamaguchi, K. Namba and S. Aizawa (1992). "Morphological 445

446

447 pathway of flagellar assembly in Salmonella typhimurium." \ Mol Biol 226(2): 433-446.

Kudva, R., K. Denks, P. Kuhn, A. Vogt, M. Muller and H. G. Koch (2013). "Protein translocation across the inner membrane of Gram-negative bacteria: the Sec and Tat dependent protein transport pathways." Res Microbiol 164(6): 505-534.

450 Leaver-Fay, A., M. Tyka, S. M. Lewis, O. F. Lange, J. Thompson, R. Jacak, K. Kaufman, P. D. Renfrew, C. A. Smith, W. Sheffler, I. W. Davis, S. Cooper, A. Treuille, D. J. Mandell, F. Richter, Y. E. Ban, S. J. Fleishman, J. E. Corn, D. E. Kim, S. Lyskov, M. Berrondo, S. Mentzer, Z. Popovic, 452 J. J. Havranek, J. Karanicolas, R. Das, J. Meiler, T. Kortemme, J. J. Gray, B. Kuhlman, D. Baker 453 and P. Bradley (2011). "ROSETTA3: an object-oriented software suite for the simulation and design of macromolecules." Methods Enzymol 487: 545-574.

455 Levdikov, V. M., E. V. Blagova, A. McFeat, M. J. Fogg, K. S. Wilson and A. J. Wilkinson (2012). "Structure of components of an intercellular channel complex in sporulating Bacillus subtilis." Proc Natl Acad Sci U S A 109(14): 5441-5445.

458 Levenson, R., H. Zhou and F. W. Dahlquist (2012). "Structural insights into the interaction between the bacterial flagellar motor proteins FliF and FliG." Biochemistry 51(25): 5052-

4605060.

461 Li, W., A. Cowley, M. Uludag, T. Gur, H. McWilliam, S. Squizzato, Y. M. Park, N. Buso and R. 462 Lopez (2015). "The EMBL-EBI bioinformatics web and programmatic tools framework." Nucleic Acids Res 43(W1): W580-584.

464 McWilliam, H., W. Li, M. Uludag, S. Squizzato, Y. M. Park, N. Buso, A. P. Cowley and R. Lopez 465 (2013). "Analysis Tool Web Services from the EMBL-EBI." Nucleic Acids Res 41(Web 466 Server issue): W597-600.

467 Meisner, J., T. Maehigashi, I. Andre, C. M. Dunham and C. P. Moran, Jr. (2012). "Structure of 468 the basal components of a bacterial transporter." Proc Natl Acad Sci U S A 109(14): 54464695451.

470 Meisner, J., X. Wang, M. Serrano, A. O. Henriques and C. P. Moran, Jr. (2008). "A channel 471 connecting the mother cell and forespore during bacterial endospore formation." Proc Natl 472 Acad Sci U S A 105(39): 15100-15105. 
473 Minamino, T. and K. Imada (2015). "The bacterial flagellar motor and its structural 474 diversity." Trends Microbiol 23(5): 267-274.

475 Minamino, T., K. Imada, M. Kinoshita, S. Nakamura, Y. V. Morimoto and K. Namba (2011). 476 "Structural insight into the rotational switching mechanism of the bacterial flagellar 477 motor." PLoS Biol 9(5): e1000616.

478 Minamino, T., K. Imada and K. Namba (2008). "Mechanisms of type III protein export for 479

480

481

482

483

484

485

486

487

488

489

490

491

492

493

494

495

496

497

498

499

500

501

502

503

504

505

506

507

508

509

510

511

512

513

514

515

516

517 bacterial flagellar assembly." Mol Biosyst 4(11): 1105-1115.

Morimoto, Y. V. and T. Minamino (2014). "Structure and function of the bi-directional bacterial flagellar motor." Biomolecules 4(1): 217-234.

Nans, A., M. Kudryashev, H. R. Saibil and R. D. Hayward (2015). "Structure of a bacterial type III secretion system in contact with a host membrane in situ." Nat Commun 6: 10114. Notti, R. Q., S. Bhattacharya, M. Lilic and C. E. Stebbins (2015). "A common assembly module in injectisome and flagellar type III secretion sorting platforms." Nat Commun 6: 7125.

Ogawa, R., R. Abe-Yoshizumi, T. Kishi, M. Homma and S. Kojima (2015). "Interaction of the C-terminal tail of FliF with FliG from the Na+-driven flagellar motor of Vibrio alginolyticus." LBacteriol 197(1): 63-72.

Peters, J., D. P. Wilson, G. Myers, P. Timms and P. M. Bavoil (2007). "Type III secretion a la Chlamydia." Trends Microbiol 15(6): 241-251.

Petersen, T. N., S. Brunak, G. von Heijne and H. Nielsen (2011). "SignalP 4.0: discriminating signal peptides from transmembrane regions." Nat Methods 8(10): 785-786.

Pruitt, K. D., T. Tatusova, G. R. Brown and D. R. Maglott (2012). "NCBI Reference Sequences (RefSeq): current status, new features and genome annotation policy." Nucleic Acids Res 40(Database issue): D130-135.

Rohl, C. A., C. E. Strauss, K. M. Misura and D. Baker (2004). "Protein structure prediction using Rosetta." Methods Enzymol 383: 66-93.

Schraidt, O. and T. C. Marlovits (2011). "Three-dimensional model of Salmonella's needle complex at subnanometer resolution." Science 331(6021): 1192-1195.

Spreter, T., C. K. Yip, S. Sanowar, I. Andre, T. G. Kimbrough, M. Vuckovic, R. A. Pfuetzner, W. Deng, A. C. Yu, B. B. Finlay, D. Baker, S. I. Miller and N. C. Strynadka (2009). "A conserved structural motif mediates formation of the periplasmic rings in the type III secretion system." Nat Struct Mol Biol 16(5): 468-476.

Stock, D., K. Namba and L. K. Lee (2012). "Nanorotors and self-assembling macromolecular machines: the torque ring of the bacterial flagellar motor." Curr Opin Biotechnol 23(4): 545-554.

Stone, C. B., D. C. Bulir, J. D. Gilchrist, R. K. Toor and J. B. Mahony (2010). "Interactions between flagellar and type III secretion proteins in Chlamydia pneumoniae." BMC Microbiol 10: 18 .

Suzuki, H., K. Yonekura, K. Murata, T. Hirai, K. Oosawa and K. Namba (1998). "A structural feature in the central channel of the bacterial flagellar FliF ring complex is implicated in type III protein export." I Struct Biol 124(2-3): 104-114.

Suzuki, H., K. Yonekura and K. Namba (2004). "Structure of the rotor of the bacterial flagellar motor revealed by electron cryomicroscopy and single-particle image analysis." I Mol Biol 337(1): 105-113.

Terashima, H., S. Kojima and M. Homma (2008). "Flagellar motility in bacteria structure and function of flagellar motor." Int Rev Cell Mol Biol 270: 39-85. 
518 Thomas, D., D. G. Morgan and D. J. DeRosier (2001). "Structures of bacterial flagellar motors 519 from two FliF-FliG gene fusion mutants." ¿ Bacteriol 183(21): 6404-6412.

520 Thomas, D. R., N. R. Francis, C. Xu and D. J. DeRosier (2006). "The three-dimensional 521 structure of the flagellar rotor from a clockwise-locked mutant of Salmonella enterica 522 serovar Typhimurium." Journal of bacteriology 188(20): 7039-7048.

523 Ueno, T., K. Oosawa and S. Aizawa (1994). "Domain structures of the MS ring component 524 protein (FliF) of the flagellar basal body of Salmonella typhimurium." L Mol Biol 236(2): 525 546-555.

526 Yip, C. K., T. G. Kimbrough, H. B. Felise, M. Vuckovic, N. A. Thomas, R. A. Pfuetzner, E. A. Frey, 527 B. B. Finlay, S. I. Miller and N. C. Strynadka (2005). "Structural characterization of the 528 molecular platform for type III secretion system assembly." Nature 435(7042): 702-707. 


\section{Figure $\mathbf{1}$ (on next page)}

The flagellar, T3SS and sporulation complexes.

Schematic representation of the bacterial flagellum (A), the T3SS (B), and the sporulation complex (C). Sct-like components are in blue, SctD-like components in green, and outermembrane components in yellow. The EM maps are shown in grey for (A) and (B). The ring structures identified in the flagellum are also indicated. IM is for inner membrane, OM is for outer membrane, IFM is for inner forespore membrane, OFM is for outer forespore membrane. 


\section{Figure 2 (on next page)}

Domain organization of FliF.

Multiple sequence alignment of FliF sequences from various human pathogens (Salmonella typhimurium, Escherichia coli, Yersinia pestis, Bordetella pertussis, Pseudomonas aeruginosa, Legionella pneumophilia, Helicobacter pylori, Campylobacter jejuni, Listeria monocytogenes, Streptococcus pneumonia, Vibrio cholerae, Bacillus subtilis, Clostridium difficile, Treponema palladium). Conserved residues are in red box, similar residues are in red characters. Identified domains are highlighted in colored boxes, with the TM helices in yellow, the FliGbinding domain in green, the signal sequence in purple, the Sct] homology domain in blue and the FliF-specific domain in orange. The predicted secondary structure elements for the $S$. typhimurium FliF are in blue at the top. 


\section{Figure 3 (on next page)}

The Chlamydia FliF orthologue has unusual domain architecture.

(A) Multiple sequence alignment of FliF sequences from the Chlamydiacae family ( $C$.

trachomatis, C. muridarum, C. suis from the genus Chlamydia, and C. psittaci, C. abortus, C. felis, C. caviae, C. ibidis, C. pneumonia, and C. pecorum from the genus Chlamydophila. Labeling is as in figure $A$, with the secondary structure prediction of the $C$. trachomatis orthologue shown at the top. (B) Schematic representation of FliF and its interaction with FliG (top), and of the Chlamydia FliF-FliG fusion (bottom). 
Figure 4(on next page)

Modeling of the S. typhimurium FliF PrgK homology domain.

(A) Sequence alignment of the periplasmid domains from the EPEC and S. typhimurium Sct) homologues, EscJ and PrgK, with that of the SctJ homology domain of FliF (residues 50-221). Secondary structure elements for EscJ (PDB ID: 1YJ7) and PrgK (PDB ID: 3J6D) are shown at the top, in blue and green respectively. (B) and (C) Energy plot for the refinement of the FliF RBM1 and RBM2. The RMSD values are computed for all atoms, relative to the lowest-energy model. (D) and (E) Cartoon representation of the lowest-energy models for the Flif RBM1 and RBM2, with rainbow coloring indicating $\mathrm{N}$ - to $\mathrm{C}$-termini. 
Figure $\mathbf{5}$ (on next page)

Modeling of the FliF-specific domain.

(A) Sequence alignment of the EscJ RBM2, PrgK RBM2, SpolllAG and SpolllAH, with that of the FliF-specific domain (residues 228-439). Secondary structure elements for EsC) (PDB ID: 1YJ7) and SpollIAH (PDB ID: 3UZ0) are shown at the top, in blue and green respectively. The location of the insert ion FliF and SpolllAG is indicated. (B) Energy plot for the refinement of the FliF RBM3. The RMSD values are computed for all atoms, relative to the lowest-energy model. (C) Cartoon representation of the lowest-energy model for the FliF RBM3, with rainbow coloring indicating $\mathrm{N}$ - to $\mathrm{C}$-termini. The location of the insert is indicated. 

ecececelecelecee $\quad$ T... T $\stackrel{\beta 4}{\longrightarrow}$

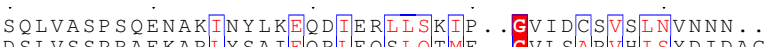

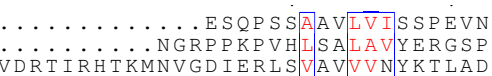

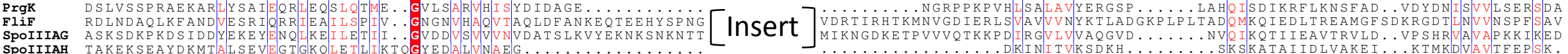

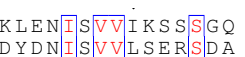

B

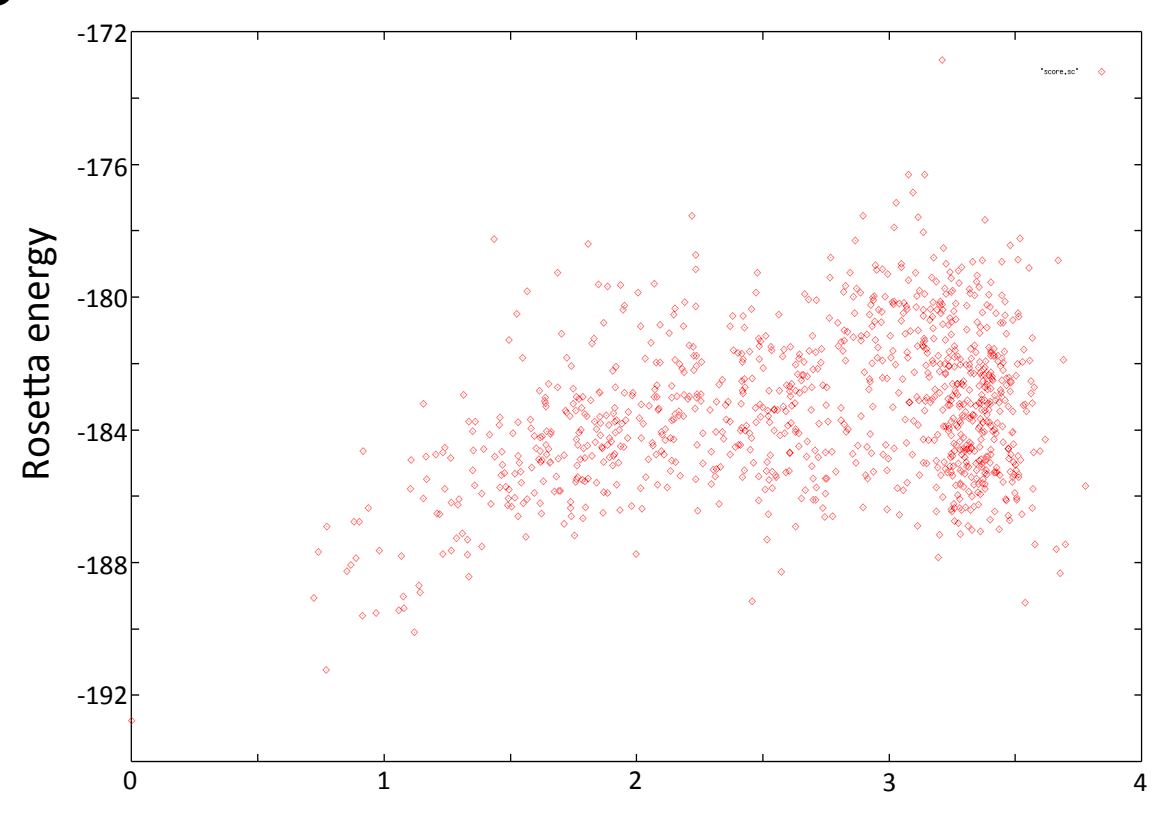

C

Insert

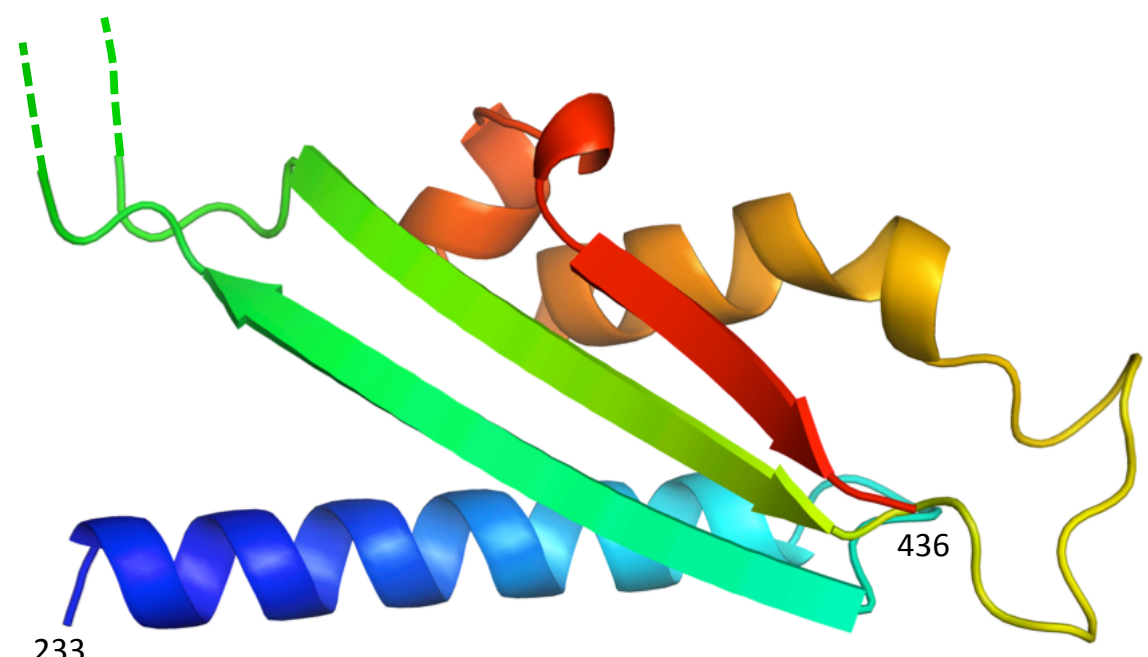

233 
Figure $\mathbf{6}$ (on next page)

Comparison of the flagellar and T3SS basal body.

(A) EM maps of the S. typhimurium T3SS basal body (EMBD ID: 1875) in black, overlaid on that of the $S$. typhimurium flagellum basal body (EMBD ID: 1887) in yellow. A close-up view of the S-ring region of the flagellum is shown on the left, with the Sct)/SctD ring model (PDB ID 3J6D) docked in the T3SS EM map. The SctJ RBM1 is in cyan and the RBM2 in blue, and the three SctD RBMs are in green. (B) Schematic representation of SctJ, SctD and FliF, with the TMs in yellow, and the RBMs colored as in (A) for SctJ and SctD. Corresponding domains are indicated in FliF. 


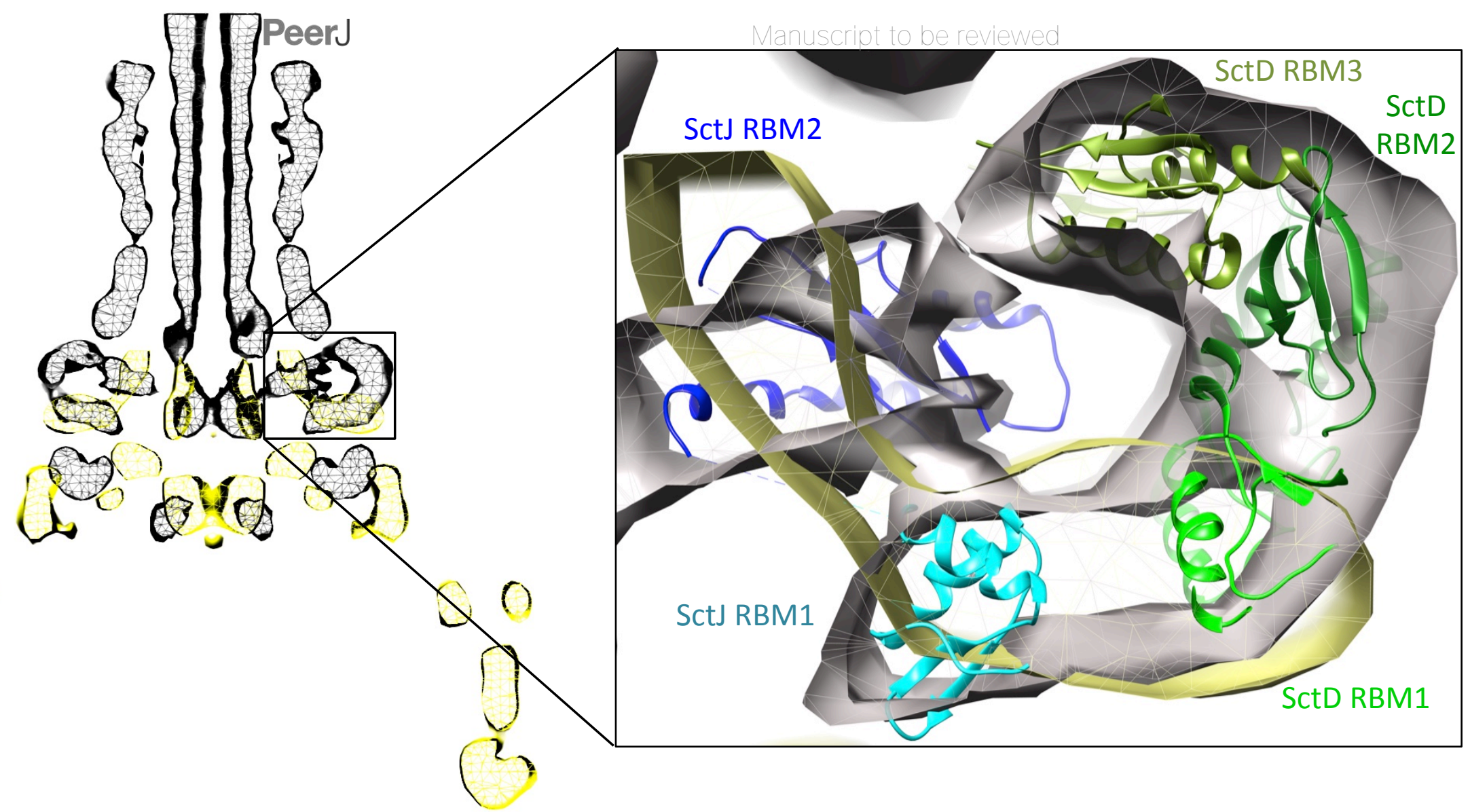

B

SctJ

\begin{tabular}{|c|c|c|c|}
\hline RBM1 & RBM2 & TM2 & Cytosolic tail \\
\hline
\end{tabular}

SctD

Cytosolic domain

TM1

RBM1

RBM2

RBM3

TM1

RBM1

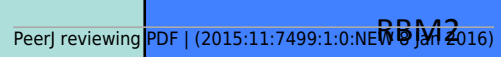

RBM3

TM2

FliG-binding domain 
Figure 7 (on next page)

Modeling of the FliF oligomer.

(A) Docking of the three FliF RBM models in the FliF EM map. The domains are colored as in figure 5B. (B) Energy plot for the EM-guided symmetry docking procedure of the FliF RBM3. The RMSDs are computed for backbone atoms of the entire modeled $24 \mathrm{mer}$ complex, relative to the lowest-energy model, and color-coded depending on the fit to EM map. Three clusters of low-energy models were identified $(\mathrm{Cl}-1, \mathrm{Cl}-2$ and $\mathrm{Cl}-3)$, with two adjacent molecules for each cluster shown in (C). The 25-mer radius axis is represented by a dotted line. (D) 25-mer model of the Flif periplasmic region, viewed from the top. 
$A$
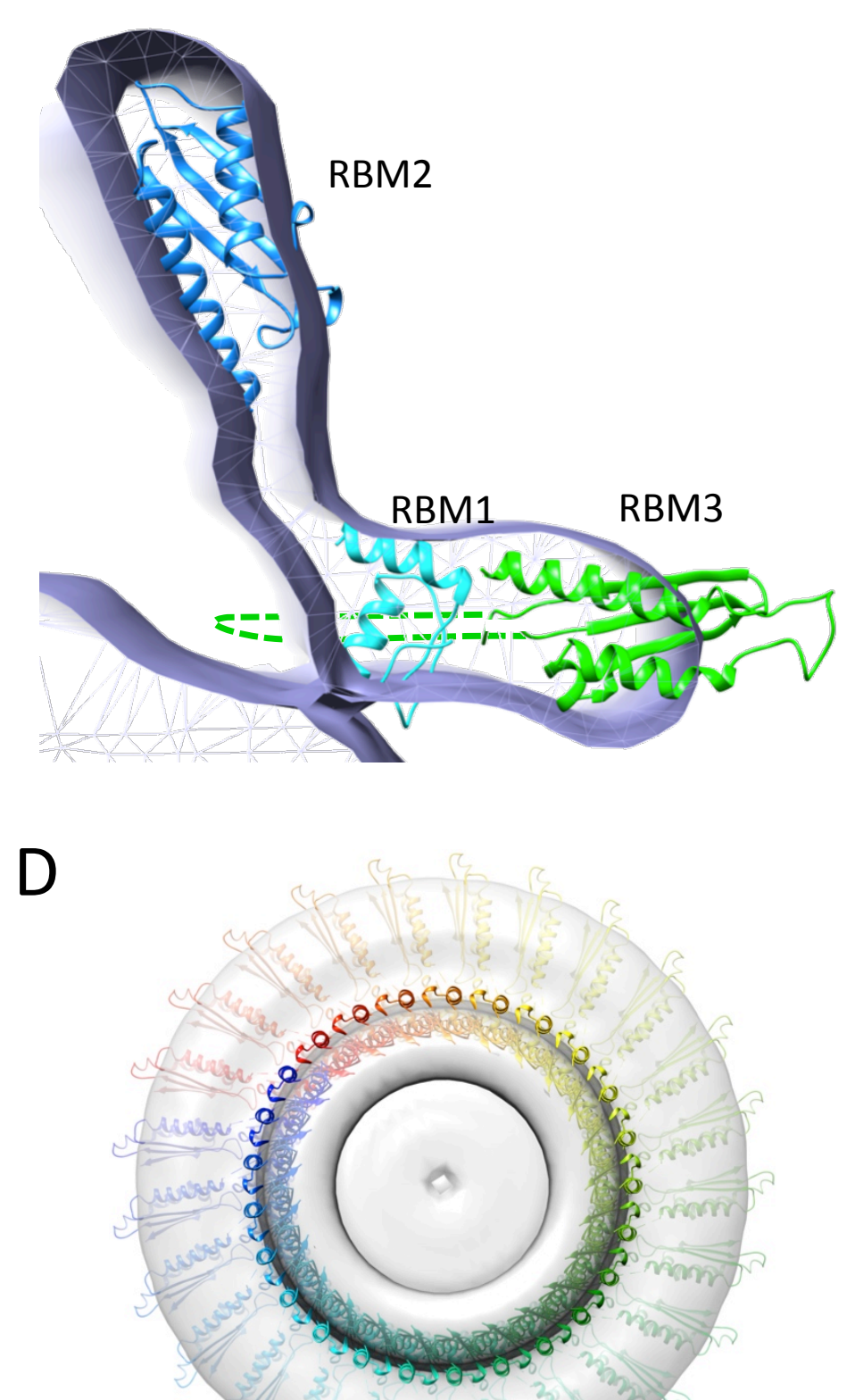

B

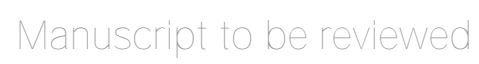

EM score

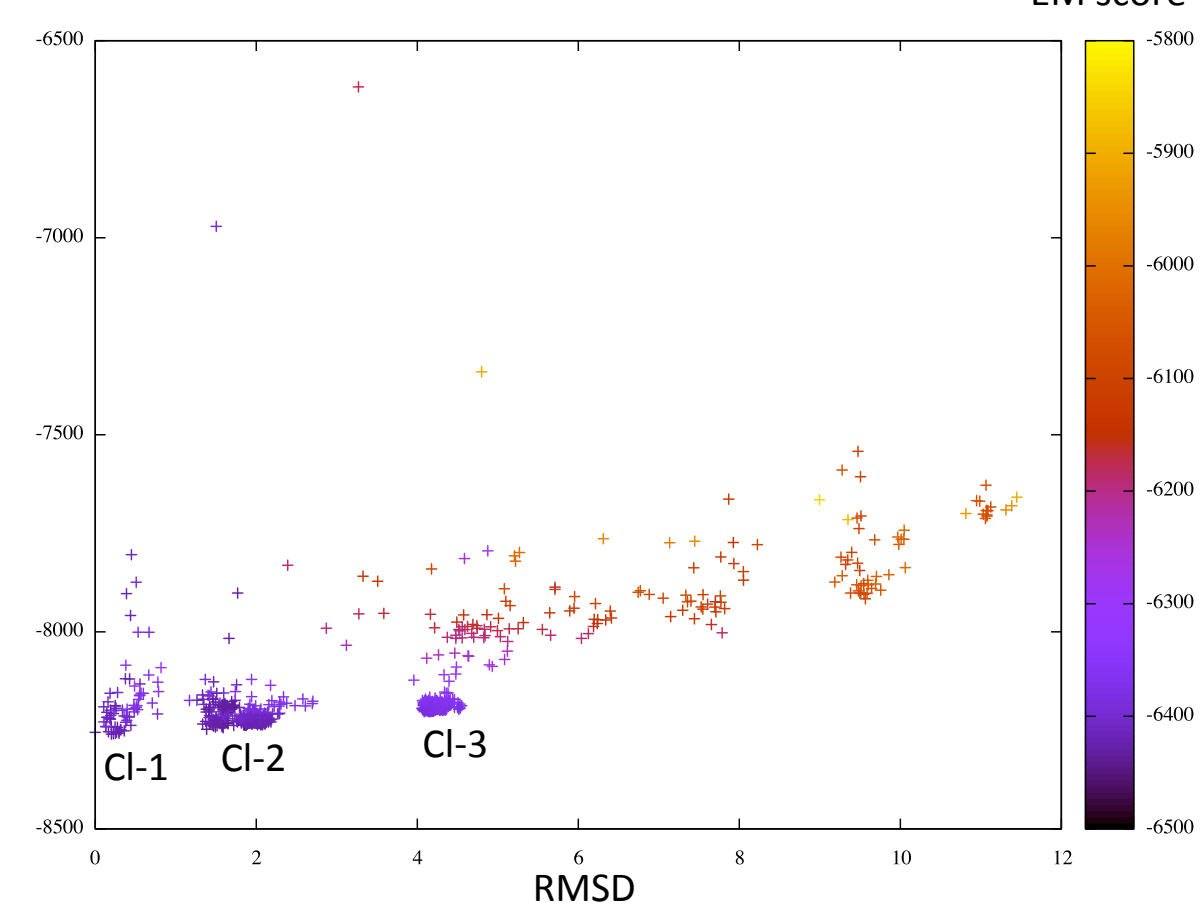

C
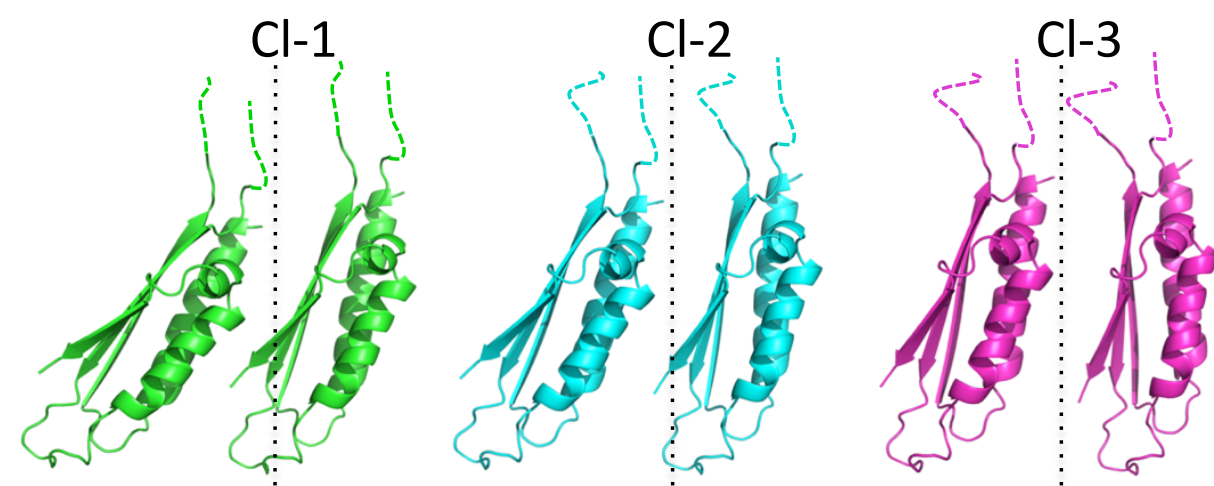


\section{Table $\mathbf{1}$ (on next page)}

Sequence identity between FliF orthologues used in the multiple sequence alignment shown in Figure 2.

The sequence identity between each pair, as calculated with the Needleman-Wunsch algorithm. 


\begin{tabular}{|c|c|c|c|c|c|c|c|c|c|c|c|c|c|c|}
\hline & Salmonella & E.coli & Yersinia & Bordetella & $\begin{array}{c}\text { Pseudomona } \\
\mathrm{s}\end{array}$ & Legionella & Helicobacter & Campylobacter & Listeria & Streptococcus & Vibrio & Bacillus & Clostridium & Treponema \\
\hline Salmonella & 100.0 & 85.9 & 62.4 & 45.1 & 34.1 & 34.7 & 30.7 & 29.4 & 23.6 & 21.7 & 27.9 & 24.6 & 26.2 & 21.5 \\
\hline E.coli & & 100.0 & 62.6 & 46.2 & 33.7 & 34.7 & 31.4 & 29.6 & 22.0 & 22.0 & 27.7 & 25.1 & 25.0 & 22.2 \\
\hline Yersinia & & & 100.0 & 49.2 & 35.5 & 34.5 & 29.6 & 28.1 & 21.2 & 21.1 & 26.6 & 24.2 & 23.9 & 19.5 \\
\hline Bordetella & & & & 100.0 & 35.9 & 35.1 & 29.6 & 26.3 & 22.9 & 21.0 & 27.5 & 23.2 & 22.0 & 21.6 \\
\hline Pseudomonas & & & & & 100.0 & 38.1 & 28.4 & 29.0 & 20.3 & 20.3 & 31.0 & 20.0 & 22.2 & 20.3 \\
\hline Legionella & & & & & & 100.0 & 28.8 & 26.5 & 20.2 & 20.2 & 29.3 & 21.2 & 25.3 & 20.2 \\
\hline Helicobacter & & & & & & & 100.0 & 43.2 & 23.7 & 21.9 & 23.3 & 23.8 & 28.3 & 24.2 \\
\hline Campylobacter & & & & & & & & 100.0 & 22.8 & 21.2 & 26.1 & 23.1 & 23.3 & 25.0 \\
\hline Listeria & & & & & & & & & 100.0 & 37.4 & 19.8 & 22.3 & 24.5 & 20.5 \\
\hline Streptococcus & & & & & & & & & & 100.0 & 20.5 & 22.4 & 24.4 & 21.3 \\
\hline Vibrio & & & & & & & & & & & 100.0 & 19.1 & 21.8 & 18.6 \\
\hline Bacillus & & & & & & & & & & & & 100.0 & 24.6 & 23.2 \\
\hline Clostridium & & & & & & & & & & & & & 100.0 & 20.7 \\
\hline Treponema & & & & & & & & & & & & & & 100.0 \\
\hline
\end{tabular}




\section{Table 2 (on next page)}

Geometry validation scores for the structural models of the three Flif RBMs.

Geometry parameters for each atomic models, obtained with the Protein Structure Validation Suite. 


\begin{tabular}{|l|c|c|c|}
\hline & RBM1 & RBM2 & RBM3 \\
\hline Ramachandran favored (\%) & 98.0 & 97.8 & 98.9 \\
\hline Ramachandran allowed (\%) & 0.0 & 1.1 & 1.1 \\
\hline Ramachandran disallowed (\%) & 2.0 & 1.1 & 0.0 \\
\hline Verify3D score & 0.46 & 0.38 & 0.31 \\
\hline Procheck score & 0.39 & 0.26 & 0.20 \\
\hline MolProbity Clashscore & 1.25 & 4.22 & 13.10 \\
\hline Close contacts & 0 & 0 & 0 \\
\hline RMSD bond angle ( $(\AA)$ & 1.5 & 2.6 & 1.8 \\
\hline RMSD bond length ( $\left.{ }^{\circ}\right)$ & 0.010 & 0.013 & 0.023 \\
\hline
\end{tabular}

1 